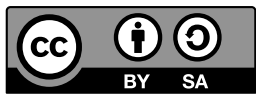

\title{
El acto discursivo y la negociación de los significados léxicos en el contexto (inter)cultural de aprendizaje de español como lengua extranjera
}

Palabras clave: discurso, negociación, significado, léxico, comunicación intercultural.

\section{El discurso y el contexto cultural}

El discurso es un concepto multidisciplinar o transdisciplinar, analizado desde los años setenta por varias ciencias: la lingüística (analiza la lengua y su forma de uso), la psicología (analiza las convicciones de los miembros de una sociedad y el modo de su expresión), las ciencias sociales (analizan las interacciones en contextos sociales), etc. Estamos de acuerdo con van Dijk (2001: 21) en que la noción de discurso es «esencialmente difusa» porque remite a fenómenos bastante complejos relacionados sobre todo con la comunicación humana, o mejor dicho, con la interacción verbal. En opinión de Kurcz (2005), el discurso tiene carácter oral (p. ej. la conversación) y el texto pertenece a la transmisión escrita (p. ej. el libro, la carta, el contrato, etc.). En cambio, van Dijk (2001: 23) considera que también los textos escritos (excluyendo los libros, las leyes, las publicaciones de carácter académico o las cartas ${ }^{1}$ ) deberían formar parte del discurso, ya que «hay mucha similitud en la manera como las personas hablan o escriben cuando utilizan el lenguaje para comunicar sus ideas y lo mismo ocurre cuando la gente escucha o lee un discurso».

Coseriu (1981: 272) denomina el discurso como «acto lingüístico - o la serie de los actos lingüísticos conexos - de un individuo determinado en una situación

1 van Dijk (2001:24) opina que las cartas, los libros, etc. «parecen ser objetos, o productos de actos verbales, más que formas de interacción». 
determinada». En un acto lingüístico aparecen las expresiones lingüísticas (el significado) y las situaciones, personas, etc. (lo designado) que se convierten en instrumentos que sirven para expresar un determinado sentido². El discurso está unido a una manifestación verbal de la lengua que es dominante en el conjunto de las manifestaciones lingüísticas y también corresponde a cierto conjunto de signos formales (Charaudeau, 1984). Lo mismo pasa con el código de gestos (comunicación no verbal) o código iconográfico (lengua de imagen). El discurso en este sentido no es solamente un texto, una frase, una palabra, sino también un gesto, la tonalidad de la voz, etc. En otras palabras, no existe un acto comunicativo suelto, o sea, desligado de su situación contextual ${ }^{3}$.

De lo expuesto hasta aquí se desprende que el discurso funciona como la lengua dentro de un contexto concreto (van Dijk, 2011). Siendo una forma del uso de la lengua (hablada o escrita), hay que analizar quién usa una determinada forma lingüística, cómo, por qué y cuándo (van Dijk, 2001)4. Por ejemplo, Kramsch (1998) opina que ser miembro de una cultura significa pertenecer al discourse community con su historia y su sistema común, lo que permite actuar de una y no de otra manera. La comunidad discursiva dispone de su propio registro de normas comunicativas (véase Duszak, 2001).

Sánchez Lobato (1999: 7) argumenta que la cultura establece normas de orientación que clasifican los tipos de pautas, creencias, símbolos y orientaciones de valor que posibilitan el desarrollo de la comunicación. Así, estas normas favorecen la coparticipación y concretan significados que pueden considerarse relativamente estables y que son expresiones de expectativas comunes. En su opinión, la lengua forma parte del sistema cultural y adquiere significado propio como una expectativa de comportamientos compartidos, como un conjunto de técnicas, prácticas de comunicación y estructuras lingüísticas que son parte del conocimiento social transmitido y heredado a través de los procesos de socialización lingüísticos. La cultura, por tanto, supone un proceso de interacción entre los seres humanos, de significados compartidos, que tiende a la configuración de los sistemas simbólicos.

2 Podemos hablar entonces de un discurso político, médico, religioso, etc. Por ejemplo, los médicos de diferentes naciones pueden comunicarse a través del mismo discurso.

3 Charaudeau (1984), en el ámbito de su teoría analítico-discursiva, elaboró un modelo dividido en tres niveles, donde cada nivel corresponde a un tipo de sujeto: el nivel situacional relacionado con la competencia situacional; el nivel discursivo relacionado con la competencia discursiva, y nivel sociolingüístico relacionado con la competencia sociolingüística.

4 Por ejemplo, en la teoría de la relevancia (Grice, 1972; Sperber y Wilson, 1986/1995) también se aconseja obtener del entorno físico (incluyendo los enunciados verbales y no verbales), información sobre las intenciones comunicativas de los hablantes. 
La unión entre la cultura y el discurso es incuestionable, sobre todo cuando se analizan las expresiones de la vida cotidiana. Seguidamente aparecen algunos ejemplos de expresiones propias de la lengua y cultura españolas que demuestran que al disponer de unas pautas culturales se exige el uso de determinados comportamientos comunicativos o registros verbales:

1. No te molestes, esto lo pago yo [Una muestra de negociación del pago habitual en España.]

2. Vivo en Castellana, 25, $2^{\circ}, 3$ [Una forma habitual de dar la dirección: calle, número, piso, puerta.]

3. ¿Por qué no nos tuteamos? [El tuteo es algo natural en España, considerando las diferentes reglas contextuales.]

4. Esta es Eva, la madrina de mi hijo. Eva es la novia de mi hermano mayor. [A1 presentar a una persona damos una explicación de qué tipo de conexión hay entre nosotros.]

(tomado de Miquel y Sans, 1992: 17).

Resumiendo, el discurso en su sentido global funciona como el producto de una determinada comunidad discursiva, implica la presencia del contexto y la cultura durante un acto comunicativo que se realiza a través de una interacción que también puede tener características de negociación.

\section{La negociación como tipo discursivo}

La negociación es un acto comunicativo, conversacional, es decir, es un tipo discursivo que puede ser realizado en varios ámbitos tanto personales como profesionales. Dolón Herrero (1998: 2) señala que es un fenómeno que «por un lado se puede dar en prácticamente cualquier entorno socio-cultural, y por otro lado se constituye a partir de importantes variables del contexto inmediato de negociación». Entre las variables del contexto la autora menciona a los participantes (número de negociadores, su experiencia, rol que representan), la relación que hay entre ellos, la complejidad de los puntos a tratar y la ubicación espacio-temporal.

La negociación también depende de la comunicación entre los individuos que representan distintos componentes conductuales, necesidades o deseos (Nierenberg, 1994). Kamiński (2004: 16) define la negociación como un proceso en el cual se resuelve un conflicto. Así pues, dos o más partes discuten sobre 
las diferencias que les separan y tratan de tomar una decisión en cuanto a los asuntos para ellos más importantes5. Otros autores, como Costa García, Galeote Muñoz y Segura Gálvez (2004: 13), tratan de establecer una definición que abarque todas las características básicas del proceso de negociación, diciendo que: «es un proceso de interacción en el que las partes involucradas, conjugando mecanismos de influencia y persuasión, persiguen alcanzar un acuerdo que satisfaga de forma equitativa sus respectivos intereses».

El objetivo principal de las negociaciones es llegar a un acuerdo basado especialmente en una comunicación cooperativa o colaborativa en términos de que todos ganan 6 . Se trata de buscar «ventajas mutuas siempre que sea posible, y que cuando haya conflicto de intereses debe insistirse en que el resultado se base en algún criterio justo, independientemente de la voluntad de las partes» (Fisher, Ury, Patton, 2004: 29), lo que, sin duda, requiere el conocimiento mutuo de las personas implicadas en la negociación. También hay que tener en cuenta que «una forma de brindar el reconocimiento al otro es reconocer su punto de vista, sin tener necesariamente que concordar con él» (Ury, 2007: 88).

Es evidente que en la negociación intervienen factores tanto extralingüísticos como lingüísticos. Los dos tipos se unen de manera directa con la pragmática, que estudia los principios que regulan el uso del lenguaje de la comunicación, «es decir, las condiciones que determinan el empleo de enunciados concretos emitidos por hablantes concretos en situaciones comunicativas concretas, y su interpretación por parte de los destinatarios» (Escandell Vidal, 1993: 16). La negociación como acto discursivo se realiza también mediante la conversación (formal e informal o coloquial) en la que las intervenciones dependen de las circunstancias y las características de los interlocutores.

a) Conversar es argumentar, aportar (dar) argumentos con un fin concreto (todo acto comunicativo tiene una intención, que coincide con lo dicho o ha de interpretarse).

b) Y a la vez es negociar el acuerdo; es interactuar, mantener una relación cooperativa, regulada además por una serie de normas de conducta social, las cuales, en ocasiones, se ven alteradas por principios

5 Ovejero Bernal (2004) añade que el conflicto puede ser visto también como algo sociológicamente necesario que puede provocar cambios muy positivos tanto a nivel individual como general. El autor subraya que la negociación, a diferencia de la imposición, es la forma más eficaz de solucionar los problemas.

6 Es un método de negociación según los principios descritos por Ury, Fisher y Patton (2004) y desarrollado en el Proyecto de la Negociación de Harvard. También existen negociaciones llamadas posicionales en las que siempre hay un perdedor. 
de pertinencia comunicativa, de adecuación a la situación, alteraciones muchas veces estratégicas

(Briz Gómez, 2011: 51-52).

Otra visión de las negociaciones es la que presenta Gómez de Enterría Sánchez (2009), quien opina que este acto discursivo forma parte de las lenguas de especialidad. Estas se diferencian de la lengua común por la terminología, el léxico especializado y los elementos lingüísticos que se utilizan durante la conversación o discusión. Entre estos últimos aparecen: los argumentos, las paráfrasis, las expresiones modalizadoras y las oposiciones. Los argumentos sirven para convencer, persuadir y mantener una discusión abierta. «Argumentar significa un esfuerzo para apoyar un punto de vista con razones» (Weston, 2012: 13). Por otra parte, las repeticiones o las paráfrasis pueden reforzar y organizar el discurso argumentativo recordando los puntos clave de la conversación. La paráfrasis es un recurso que sirve para reformular o aclarar ideas o expresiones que resultan poco claras o comprensibles. «Suelen ir introducidas por los reformuladores argumentativos: es decir, o sea, esto es, etc.» (Gómez de Enterría Sánchez, 2009: 140). En cuanto a las expresiones modalizadores, hay que recordar, que su principal función consiste en llamar la atención del interlocutor o expresar la propia opinión:

1. ...permitame que le diga...

2. ...si me permite, yo se lo voy a explicar a usted

3. .... mi modo de ver...

4. ...desde mi punto de vista...

(Gómez de Enterría Sánchez, 2009: 141). En cambio, las oposiciones sirven para resaltar una idea a través del contraste con otros conceptos antes mencionados. En estas situaciones se usa a menudo las estructuras interrogativas:

5. ...ino le parece a usted?

6. ...ino cree usted?

7. ino es cierto que?...

(Gómez de Enterría Sánchez, 2009: 141).

Cada negociación contiene también distintos contenidos comunicativos que se corresponden con los actos de babla. Los más destacados son los actos 
locutivos, proposicionales e ilocutivos definidos por Austin (1982). Los actos locutivos son unos enunciados con un significado concreto realizados por medio de una secuencia de sonidos. Entre ellos destacan las preguntas que parecen cruciales en la negociación de los significados. Tannen (2002) recuerda que las preguntas pueden ayudar en la clarificación de lo que se dice (el mensaje). No obstante, también pueden funcionar en el nivel del metamensaje (lo que realmente queremos decir). Por ejemplo, en vez de decir ¡No lo hagas! (mensaje) decimos ¿Por qué lo baces? Los mencionados antes actos proposicionales (o de predicción) hacen referencia al hecho de referir y predicar. Y los actos ilocutivos expresan la intención de los hablantes (Escandell Vidal, 2014: 95)7.

Por su parte Searle $(1975,1990)$ distinguió los actos directos (asertivos, directivos, compromisivos, declarativos, expresivos), indirectos (el significado no coincide con la intención) y realizativos. Abajo presentamos algunos ejemplos:

8. Estálloviendo. (acto asertivo)

9. Explícamelo. (acto directivo)

10. Este fin de semana os voy a visitar. (acto compromisivo)

11. ¡Os declaro marido y mujer! (acto declarativo)

12. No entiendo nada. (acto expresivo)

13. Tengo que escribirle un mensaje. (acto realizativo)

14. ¿Quieres otro café? (acto indirecto, puede significar que el invitado debería irse ya a casa) $)^{8}$.

Parece que en la comunicación intercultural los más problemáticos son los actos indirectos que expresan significados implícitos tanto lingüísticos como culturales entendidos de manera equivocada. Uno de ellos es el uso de los pronombres tú, usted.

Oliveras (2000) describe varios malentendidos de carácter pragmático y contextual que prueban que si los interlocutores supieran cómo negociar los significados seguramente podrían evitar muchos sentimientos negativos y

7 Cabe mencionar que, aunque existan varias clasificaciones de los actos de habla, en cada una de ellas se subraya que ningún acto de habla puede funcionar por separado. También existe una relación entre fuerza ilocutiva y forma lingüística (Escandel Vidal, 1993).

8 En Cuba, si estamos invitados a una casa y en un momento el anfitrión ofrece un segundo café, esto puede significar que lo que quiere verdaderamente decir, es que terminemos la visita (Pons Bordería, 2005: 33). 
comunicarse de una manera mucho más eficaz. El primer malentendido tuvo lugar en España entre una chica italiana y una señora mayor que vendía periódicos en un quiosco. La chica se sentía incomoda porque la señora siempre la trataba de forma tú en vez de usted y la italiana lo consideraba como un trato inadecuado y poco respetuoso. Está claro que la señora no tenía intención de ofenderla, simplemente quería reducir la distancia y ser amable porque se trataba de una persona ya conocida por ella. Otro ejemplo de malentendido se refiere a la distinta interpretación de la palabra fiesta para una japonesa y unos españoles. La chica japonesa entendía la palabra fiesta como algo muy informal con pocas personas. Debido al poco espacio en una casa japonesa, el evento familiar se celebra en los restaurantes. En cambio, los españoles «consideraron que se trataba de una fiesta como ellos concebían: con muchas personas y comida para todos» en casa (Oliveras, 2000: 74).

A modo de conclusión, estamos de acuerdo con Oliveras (2000: 74) en que, para evitar este tipo de malentendidos, los interlocutores deberían comprobar desde principio si entienden la palabra o los enunciados de la misma manera, por ejemplo, formulando preguntas adicionales o paráfrasis ${ }^{9}$ que forman parte de la actividad negociadora. La negociación en términos muy generales es un acto comunicativo en el cual los interlocutores reciben los mensajes, los analizan o descodifican y reaccionan de manera apropiada dentro de un contexto cultural y social determinado. Sin duda, no es una tarea fácil ya que aparte de las reglas de la conversación se requiere también el conocimiento de otros elementos que intervienen en la comunicación interpersonal ${ }^{10}$ e intercultural (por ejemplo: entonación, ritmo de la voz, gestos, posturas, etc.).

9 En este punto, nos gustaría presentar un malentendido que tuvo lugar entre una amiga polaca y un amigo español. La situación tuvo lugar hace años en Madrid. Los dos eran entonces estudiantes universitarios. Un día Luis propuso a Ewa tomar un café a las diez al día siguiente y que él se acercaría a su casa. A las diez de la mañana Ewa estaba preparada para salir con Luis. Estaba segura de que Luis vendría a esa hora porque es la hora de terminar las clases o tener una pausa en las universidades polacas y asumió que era parecido en España. Pasaron las diez, las once, las doce y Luis no apareció. Luis llegó a las diez de la noche del mismo día, pero en aquel momento Ewa ya estaba en otra parte de Madrid con otros amigos. Este malentendido claramente muestra que con una pregunta de tipo ¿qué quieres decir con a las diez? Ewa no interpretaría el mensaje de manera tan equivocada.

10 Van der Hofstad Román (2003: 28-32) los divide en los componentes no verbales (expresión facial, mirada, sonrisas, postura, orientación, distancia, gestos, apariencia personal, etc. y paraverbales) y los componentes conductuales paraverbales (volumen de la voz, tono, timbre, fluidez verbal, velocidad, claridad, tiempo de habla, pausas). 


\section{La eficacia comunicativa de la negociación de los significados léxicos del mundo hispanohablante en la clase de ELE.}

El conocimiento del léxico de una lengua es fundamental en la comunicación. Si queremos transmitir un mensaje podemos prescindir de la corrección gramatical, no obstante, debemos usar las palabras adecuadas que correspondan a un determinado contexto. Además, hay que tener en cuenta que el vocabulario experimenta más cambios o modificaciones en comparación con otros componentes de la lengua como, por ejemplo, la gramática.

El Marco Común Europeo de Referencia (MCER, 2002: 108) define la competencia léxica ${ }^{11}$ como el conocimiento del vocabulario de una lengua y la capacidad para utilizarlo. Entre otros elementos léxicos el MCER (2002) distingue:

1. expresiones hechas
a. fórmulas fijas, refranes, proverbios, arcaísmos residuales,
b. modismos (metáforas, intensificadores),
c. estructuras fijas y frases hechas,

2. polisemia (una palabra puede tener varios significados).

Sin duda, el aprendizaje de una palabra nueva, aparte del conocimiento de su definición básica, abarca también los factores pragmáticos, discursivos, estilísticos, colocativos y de frecuencia (Meara, 1997). La disponibilidad léxica y la frecuencia de uso del vocabulario responden a las necesidades de las personas que aprenden una lengua extranjera. De ahí que la enseñanza del vocabulario «no puede ser una mera presentación de listas más o menos relacionadas temáticamente, sino que debe servir para diferentes actividades de aprendizaje de todo el idioma» (Romero Gualda, 2008: 21 ${ }^{12}$.

Todas estas reflexiones hacen pensar en la lengua española y su enorme variedad lingüística. ¿Es posible presentar en la clase de ELE toda la diversidad dialectal del mundo hispánico? La respuesta es obviamente negativa. Sin embargo, como dice Moreno Fernández (2010), los profesores, aunque no son

11 El MCER (2002: 112-113) distingue también la competencia semántica que comprende la conciencia y el control de la organización del significado con que cuenta el alumno: la semántica léxica, semántica gramatical y semántica pragmática.

12 No obstante, la clasificación de los temas en los que se agrupa el léxico necesario, como lo presenta por ejemplo Plan Curricular, puede ayudar a los profesores a cumplir los objetivos de la enseñanza de español en distintos niveles (PCIC, 2007). 
especialistas en la dialectología hispánica, no pueden ignorar las diferencias geolingüísticas del mundo hispanohablante. Uno de los ejemplos es el antes mencionado saludo con los tratamientos de usted, de tú, Señor, Señora y el uso de los títulos profesionales (Doctor, Licenciado, Ingeniero, etc.). ${ }^{13}$

La incomprensión discursiva a menudo surge no porque el hablante use expresiones verbales inadecuadas al contexto de saludo, sino cuando los hablantes no se dan cuenta de que los destinatarios de su mensaje desconocen el contenido sociocultural que acompaña el ritual de cortesía asociado al acto de saludar (Larionova, Romanova, 2017: 91).

Por eso, es aconsejable presentar en la clase de ELE los rasgos lingüísticos más comunes de las distintas áreas geográficas. Se trata sobre todo de afrontar los aspectos más problemáticos del mundo hispanohablante y practicar las pautas de la negociación sobre la base de varios ejemplos de palabras con distintos significados léxicos. De esta manera, se enseña a manejar las diferencias lingüísticas y culturales hasta entonces desconocidas.

A continuación, presentamos dos ejemplos de negociación de los significados léxicos del mundo hispanohablante que aparecen en nuestro manual Descubre, dedicado a los alumnos polacos que aprenden español como lengua extranjera. El objetivo es dar una idea de cómo se puede trabajar los aspectos de negociación de los significados léxicos en la clase de ELE. En el primer caso se trata de una conversación en la que aparece el problema de la errónea interpretación de las palabras comida y almuerzo $0^{14}$ por parte de dos chicos españoles y un argentino. Cuando mostraron incomprensión de los mensajes, decidieron emplear preguntas (actos locutivos) con el fin de clarificar el significado de las palabras comida y almuerzo. Los interlocutores usan también distintas fórmulas de tratamiento. El argentino no usa la forma vosotros, sino ustedes.

13 Por ejemplo, en Argentina el uso de vos o usted depende de la edad y el rango profesional. Y los títulos profesionales no son muy populares. En cambio, en México los títulos son importantes. Además, en las universidades, el trabajo u otros lugares públicos el trato es bastante formal, comparado con España donde se utiliza más el tuteo. Sin embargo, a diferencia de América Latina, en España al personal de servicios (dependientes, taxistas, camareros, etc.) se les trata de usted (Llamazares García-Lomas, 2009).

14 En varios países hispanohablantes, como Argentina, Chile, Uruguay o Colombia, se usan las palabras almuerzo y almorzar para la comida del mediodía. Y las palabras comidas o comer se usan a menudo como sinónimos de cenar (Spychała, Pascual López et al, 2016: 23) 
15. Conversación 1

Mario: ¡Qué buena está la comida!

Luis: Pues sí, está deliciosa.

Darío: ¡Muy buena está esta comida, sí! Pero yo no estoy acostumbrado a comer verdura.

Mario: ¿Nunca comes verdura?

Darío: Claro que como verdura, pero para almorzar.

Mario: ¿Verdura para almorzar? Yo en el recreo almuerzo un bocadillo $y$ mis padres tienen una pausa en el trabajo para ir a almorzar al bar, pero no comen verdura, sino un cruasán o algo así.

Darío: ¿Un cruasán para almorzar?

Luis: Sí, un cruasán y un cortado, por ejemplo.

Darío: ¡Qué raros son ustedes, los españoles! En Argentina el almuerzo es la comida más importante del día y ustedes toman dulces...

Luis: Perdona, pero la comida más importante es la comida.

Darío: ¿La comida? ¡Pero si no es bueno comer mucho por la nocbe!

Luis: ¡Cómo que por la nocbe? La comida del mediodía, digo.

Darío: O sea, el almuerzo.

Mario: A ver, que ya no sé ni de qué bablamos. ¿Qué quieres decir cuando dices "almuerzo"?

Luis: ¿Y a qué te refieres con "comida por la nocbe"?

(Spychała, Pascual López, et al., 2016: 29)

Después de leer el diálogo, proponemos a los alumnos responder a varias preguntas relacionadas con el malentendido entre Luis y Mario. Y con la ayuda de Internet los estudiantes pueden hacer un cuadro comparativo sobre el significado de las palabras almuerzo y comida en España y América Latina y qué comidas son típicas en cada lugar para el almuerzo y la comida. Este tipo de tarea desarrolla también la sensibilización intercultural.

En la Conversación 2 se negocia el significado de los horarios en España. También se explica que la palabra siesta no siempre significa dormir después de comer a mediodía. Agnieszka, de procedencia polaca, está acostumbrada 
a hacer compras en su país desde la mañana hasta la tarde porque las tiendas no suelen hacer pausas a mediodía. En cambio, en España es un poco diferente, lo que explica Carlos diciendo que, en general, no se puede hacer compras a la hora de comer. Agnieszka presenta una actitud de apertura haciendo preguntas y paráfrasis que le permiten entender mejor las diferencias culturales.

16. Conversación 2

Agnieszka: ¡ Mañana es el cumpleaños de Ana?

Carlos: ¿Ah, si? ¿Le regalamos algo?

Agnieszka: Un libro, por ejemplo.

Carlos: Vale.

Agnieszka: ¿Quedamos mañana para ir a la librería?

Carlos: ¿Mañana? Muy bien, ¿a qué bora?

Agnieszka: Por ejemplo, a las tres me viene bien.

Carlos: ¿A las tres de la tarde? Las librerías y las tiendas en general cierran desde las dos basta las cuatro.

Agnieszka: ¿Por qué cierran a esa bora?

Carlos: De dos a cuatro hacemos un descanso para comer y luego volvemos al trabajo basta bastante tarde. Si quieres comprar libros, es mejor ir de diez de la mañana a dos de la tarde o de cuatro a ocho de la tarde.

Agnieszka: Entiendo, trabajan ocho boras, como en Polonia, pero con un descanso para comer y dormir la siesta.

Carlos: A esa bora normalmente comemos, pero no todos duermen la siesta. Eso es un mito. Muchos no tienen tiempo o prefieren descansar de otra forma. Pero los centros comerciales no bacen esta pausa y abren desde la mañana basta la noche.

Agnieszka: Entonces, vamos a un centro comercial y compramos alli el libro. Carlos: De acuerdo.

(Spychała, Pascual López, et al., 2015: 57)

Los dos ejemplos de las conversaciones muestran que se puede introducir la modalidad discursiva de las negociaciones desde el principio del aprendizaje 
de la lengua extranjera. No hace falta conocer todos los rasgos y pautas de las negociaciones que se realizan entre los ejecutivos que trabajan en empresas internacionales. En una situación de extrañeza o falta de entendimiento de algunas palabras el alumno, incluso con el nivel básico de la lengua, puede usar distintos tipos de actos de habla, especialmente preguntas y paráfrasis, que le permitirán evitar y gestionar conflictos interculturales.

\section{Conclusiones}

La negociación es un fenómeno discursivo que se realiza dentro de un entorno social determinado, entre participantes que pueden representar distintas culturas nacionales. En cuanto al contexto hispanohablante hay que tener en cuenta sus distintas variaciones lingüísticas (dialectos, sociolectos, registros, etc.) y también la diversidad léxica. Es imposible conocer todas las variedades léxicas de una lengua, por eso es importante saber cómo aclarar nuevos significados que aparecen durante la actividad lingüística. Los autores del MCER (2002: 74) subrayan que, durante la conversación, el usuario de la lengua «actúa de forma alterna como hablante y oyente con uno o con más interlocutores para construir, conjuntamente, una conversación mediante la negociación de significados siguiendo el principio de cooperación». Este tipo de conducta conversacional requiere una actitud de respeto y apertura hacia las diferencias tanto lingüísticas como culturales.

En este artículo, hemos pretendido presentar un modelo de aplicación de las estrategias básicas de negociación de los significados léxicos y culturales (incluyendo el uso de determinados actos de habla), que pueden ser practicados desde el principio de aprendizaje de español como lengua extranjera. También hemos mostrado que no deberíamos vincular la negociación solamente con el mundo de los negocios o el lenguaje especializado. Se trata de ver las negociaciones como una herramienta que puede ser empleada en cualquier situación comunicativa y a la vez conflictiva.

\section{Bibliografía}

Austin, J. (1982): Cómo bacer cosas con palabras. Barcelona: Paidós.

Briz Gómez, A. (2011): El español coloquial en la conversación. Esbozo de la pragmática. Barcelona: Ariel.

Consejo de Europa (2002): Marco común europeo de referencia para las lenguas: aprendizaje, enseñanza, evaluación. Madrid: Anaya. 
Coseriu, E. (1981): Lecciones de lingüistica general. Madrid: Gredos.

Costa García, M., Galeote Muñoz, M. P., Segura Gálvez, M. (2004): Negociar para CON-vencer. Método, creatividad y persuasión en los negocios. Madrid: McGraw-Hill Interamericana.

Charaudeau, P. (1984): Langage et société, Papier de travail. Paris: Maison des Sciences de 1'Homme.

Dolón Herrero, R. (1998): La negociación como tipo discursivo. Construcción interactiva de una conducta conversacional transaccional. Valencia: Universidad de Valencia.

Duszak, A. (2001): «Języki, kultury, wspólnoty». En: Andrzej Kopczyński, Urszula Zaliwska-Okrutna (eds.), Jezyk rodzimy a jezyk obcy: komunikacja, przektad, dydaktyka, Warszawa: Wydawnictwa Uniwersytetu Warszawskiego, 7-20.

Escandell Vidal, V. M. (1993): Introducción a la pragmática. Barcelona: Antrophos.

Escandell Vidal, V. M. (2014): La comunicación. Lengua, cognición y sociedad. Madrid: Ediciones Akal.

Fisher, R., Ury, W., Patton, B. (2004): Dochodząc do TAK. Negocjowanie bez poddawania się. Warszawa: PWE.

Gómez de Enterría Sánchez, J. (2009): El español lengua de especialidad: enseñanza y aprendizaje. Madrid: Arco Libros.

Grice, H. (1975). «Logic and conversation». En: Peter Cole, Jerry Morgan (eds.), Speech Acts (Syntax and Semantics 3). Nueva York: Academic Press, 41-58.

Instituto Cervantes. (2006). Plan curricular del Instituto Cervantes. Niveles de referencia para el español. Madrid: Instituto Cervantes - Biblioteca nueva: bttp://www.cervantes.es/lengua_y_ensenanza/aprender_espanol/plan_curricular_instituto_cervantes.htm [18-09-19].

Kamiński, J. (2004): Negocjowanie. Warszawa: Poltex.

Kramsch, C. (1998): «The privilege of the intercultural speaker». En: Michael Byram, Michael Fleming (eds.), Language Learning in Intercultural Perspective. Cambridge: Cambridge University Press, 16-31.

Kurcz, I. (2005): Psychologia języka i komunikacji. Warszawa: PWN.

Llamazares García-Lomas, O. (2009): Cómo negociar con éxito en 50 países. Madrid: Global Marketing.

Larionova, M.V, Romanova, G.S. (2017): «Actos de habla en el contexto de la didáctica de ELE: ¿cómo enseñar a evitar conflictos comunicativos?’». En: Aura Luz Duffé Montalván (ed.), Singularidad y novedad en los estudios sobre los actos de babla. Madrid: Síntesis, 77-110. 
López Morales, H. (2005): La aventura del español en América. Madrid: Espasa. Meara, P. (1997): «Towards a New Approach to Modeling Vocabulary Acquisition». En: Norbert Schmitt, Michael McCarthy (eds.), Vocabulary, Description, Acquisition and Pedagogy. Cambridge: Cambridge University Press, 109-121.

Miquel, L., Sans, N. (1992): «El componente cultural: un ingrediente más de las clases de lengua». Cable, 9, 15-21.

Moreno Fernández, F. (2010): Las variedades de la lengua española y su enseñanza. Madrid: Arco Libros.

Nierenberg, G. I. (1994): Sztuka negocjacji jako metoda osiagania celu. Warszawa: Studio Emka.

Oliveras, Á. (2000): Hacia la competencia intercultural en el aprendizaje de una lengua extranjera. Madrid: Edinumen.

Ovejero Bernal, A. (2004): Técnicas de negociación. Cómo negociar eficaz y exitosamente. Madrid: McGraw Hill.

Pons Bordería, S. (2005): La enseñanza de la pragmática en la clase de E/LE. Madrid: Arcos Libros.

Romero Gualda, M. V. (2008): Léxico del español como segunda lengua: aprendizaje y enseñanza. Madrid: Arcos Libros.

Sánchez Lobato, J. (1999): Lengua y cultura en el aula de español como lengua extranjera. Madrid: SGEL.

Searle, J. R. (1975): «A Taxonomy of Illocutionary Acts». En: Keith Günderson (ed.), Language, Mind, and Knowledge, (Minneapolis Studies in the Pbilosopby of Science, vol. 7), Minneapolis: University of Minneapolis Press, 344-369.

Searle, J. R. (1990). «Expresiones, significado y actos de habla». En: John Searle (ed.): Actos de babla. Ensayos de filosofía del lenguaje. Madrid: Cátedra, 31-62.

Sperber, D., Wilson, D. (1986/1995): Relevance: Communication and Cognition. Oxford: Blackwell.

Spychała, M., Pascual López, X., Dudziak-Szukała, A., Kaźmierczak, A., García González, J. C. (2015): Descubre. Curso de español. Poziom A1 (Wydanie 1). Kraków: Wydawnictwo Draco.

Spychała, M., Pascual López, X., Dudziak-Szukała, A., Kaźmierczak, A., García González, J. C. (2016): Descubre. Curso de español. Poziom A1/A2 (Wydanie 1). Kraków: Wydawnictwo Draco.

van Dijk, Teun A. (2001): «El estudio de discurso». En: Teun A. van Dijk (ed.), El discurso como estructura y proceso. Barcelona: Gedisa, 21-66. 
van Dijk, Teun A. (2011): Sociedad y discurso. Cómo influyen los contextos sociales sobre el texto y la conversación. Barcelona: Gedisa S.A.

Van-der Hofstad, R. C. J. (2005): El libro de las babilidades de comunicación. Cómo mejorar la comunicación personal. Madrid: Ediciones Díaz de Santos, S.A.

Tannen, D. (2002): To nie tak. Jak styl konwersacyjny ksztattuje relacje $z$ innymi. Poznań: Zysk i S-ka.

Ury, W. (2007): El poder de un No positivo. Cómo decir No y sin embargo llegar a SI. Barcelona: Granica.

Weston, A. (2011): Las claves de la argumentación. Barcelona: Ariel. 
Małgorzata Spychała-Wawrzyniak

Adam Mickiewicz University of Poznań

\section{Discourse and negotiating lexical meanings in the Spanish-speaking intercultural context}

Keywords: discourse, negotiations, lexical meanings, intercultural communication

Negotiations are part of human communication. In order to communicate with other people effectively, we should also know how to react to lexical meanings that are not comprehensible within a specific social and cultural context (especially at the initial stage of a foreign language learning). Notably Spanish, for instance, is not a homogenous language but a far more complex phenomenon if we consider its dialectical varieties and thousands of cultures that represent it (López Morales, 2005). In our paper, we will present the functions, structure and characteristics of negotiations from the perspective of discourse analysis and verbal behaviours, taking into account the problems of intercultural communication in the Spanish-speaking world and teaching Spanish as a foreign language. We will also analyse conversational instruments and, in particular, speech acts, which might help learners negotiate both linguistic and cultural meanings. Finally, we will discuss models of verbal communication that might facilitate the prevention of intercultural conflicts. 
Małgorzata Spychała-Wawrzyniak

Univerza Adama Mickiewicza v Poznanju

\section{Diskurzivno dejanje in pogajanje o leksikalnih pomenih $\mathbf{v}$ (med)kulturnem kontekstu učenja španščine kot tujega jezika}

Ključne besede: diskurz, pogajanje, pomen, besedišče, medkulturno sporazumevanje

Pogajanja so del človeškega sporazumevanja. Za učinkovito sporazumevanje z drugimi pa se moramo znati odzvati na leksikalne pomene, ki jih v okviru določenega družbenega in kulturnega konteksta (še posebej na začetni ravni učenja tujega jezika) ne razumemo. Španščina na primer ni homogen jezik, temveč je zaradi narečnih različic in številčnosti kultur, ki jih zaobjema, še kako kompleksna (López Morales, 2005). V prispevku bomo predstavili vloge, strukturo in značilnosti pogajanj z vidika analize diskurza in besednega vedenja in pri tem upoštevali tudi težave medkulturnega sporazumevanja $v$ špansko govorečem svetu in pri poučevanju španščine kot tujega jezika. Analizirali bomo tudi praktičnosporazumevalna sredstva, še posebej pa govorna dejanja, ki bi učencem lahko pomagala pri pogajanju tako o jezikovnih kot o kulturnih pomenih. $\mathrm{Na}$ koncu bomo predstavili nekatere leksikalne modele besednega sporazumevanja, ki učencem lahko pomagajo pri preprečevanju medkulturnih konfliktov. 